\title{
Importance of sampling method in DNA analysis of lung cancer
}

\author{
F A Carey, D Lamb, C C Bird
}

\begin{abstract}
Lung carcinomas are characterised by considerable histological variation within the tumour. The possible effects of this morphological heterogeneity on the estimation of tumour ploidy were investigated. Multiple tissue blocks were systematically taken from 20 lung tumours and analysed by flow cytometry. The routine, archival paraffin wax embedded diagnostic blocks from these cases were also analysed. Nineteen (95\%) of the tumours were shown to contain aneuploid stemlines by systematic sampling, but if only one of these systematic tissue blocks had been taken from each case the incidence of DNA aneuploidy could have been as low as $45 \%$. Only $15(75 \%)$ tumours were aneuploid when all the routine archival blocks were analysed, but by specifically selecting tumour areas from the archival material the accuracy of this method was increased to $90 \%$.

It is concluded that tumour sampling methods are of primary importance in assessing the DNA content of lung tumours. Routine paraffin wax embedded archival tissue provides a suitable source of material for this purpose, provided that "turnover" selection is carried out.
\end{abstract}

Studies of tumour DNA content measured by flow cytometry have shown that determination of ploidy is a promising prognostic tool in non-small cell lung cancer. ${ }^{-4}$ Zimmerman et al have shown that the detection of aneuploidy in archival, formalin fixed, paraffin wax embedded tumour tissue indicates a poor prognosis, and these authors have proposed that estimation of tumour DNA content should be part of the clinical work-up of all patients with operable lung cancer. ${ }^{1}$

Lung carcinomas exhibit considerable heterogeneity of histological, ultrastructural, and immunohistochemical features within the tumours..$^{5-7}$ In a recent study we showed that this morphological variability is reflected by concomitant variation in DNA content. ${ }^{8}$ The effect of heterogeneity is that adequate tissue sampling is of the essence if a lung tumour is to be properly characterised.

\section{Methods}

Twenty consecutive lung tumour resection specimens were included in this study. Fol- lowing the routine procedure of the Department, lungs were inflated in $10 \%$ formolsaline and allowed to fix overnight. The duty pathologist, who was unaware that the case was to be included in the study, then cut the fixed specimens into slices $1 \mathrm{~cm}$ thick and selected tissue blocks for diagnosis. The lung specimens were then examined by one of the authors and the tumour was systematically sampled in the following manner (fig 1). Alternate lung slices were selected and blocks of tissue (about $1 \times 0.5 \times 0.5 \mathrm{~cm}$ ) were cut from the tumour at 12, 3, 6 and 9 o'clock and from the centre. These blocks excluded macroscopically obvious necrotic tissue. The samples were fixed in formol-saline for a further 12 hours and embedded in paraffin wax in the usual manner.

A section was cut from each of these blocks and stained with haematoxylin and eosin to confirm the presence of tumour tissue. A 50 $\mu \mathrm{m}$ section was then cut from each block and prepared for flow cytometry using a modification of the method described by Hedley et al. ${ }^{9}$ The tissue was dewaxed and rehydrated, mechanically minced, and digested for one hour at $37^{\circ} \mathrm{C}$ in a solution of $0.5 \%$ pepsin in $0.9 \% \mathrm{NaCl}$ adjusted to $\mathrm{pH} 1.5$ by adding hydrochloric acid. The suspension obtained was filtered through nylon wool and incubated overnight in propidium iodide. The samples were centrifuged before being analysed on an EPICS CS flow cytometer (Coulter, Hialeah, Florida). Immediately before analysis each sample was rinsed several times through a needle to avoid clumping of nuclei which

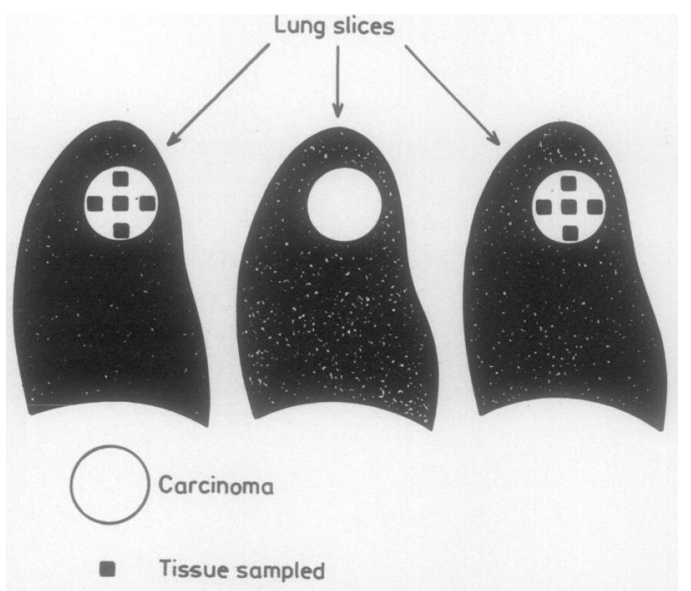

Figure 1 Tissue samples (black squares) are taken from alternate malignant lung slices from the centre of the lesion and from 12, 3, 6 and $90^{\circ}$ clock (viewed from the hilar aspect with the lung in the upright position). 
Figure 2. The

"systematic" block (left) is smaller than a typical archival block (right).

The former consists almost entirely of tumour tissue while the latter also contains normal lung (large arrow) and haemorrhagic and necrotic tissue (small arrow)

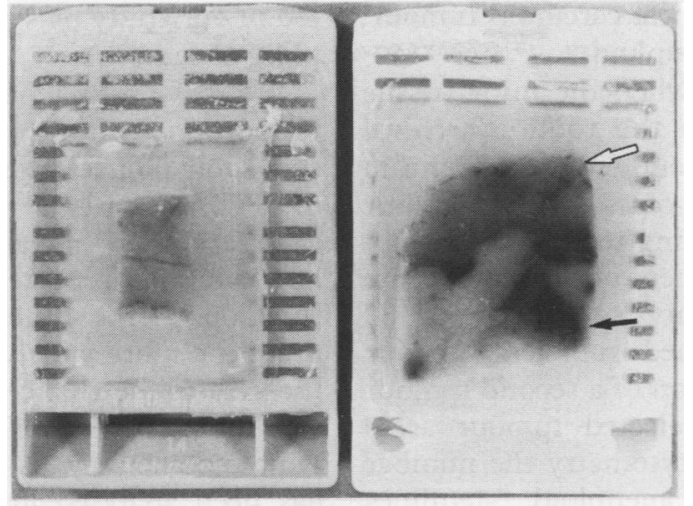

could lead to a false impression of tumour tetraploidy. Random samples were examined by fluoresence microscopy to confirm the absence of clumping. The flow cytometer is based on an argon laser tuned to $488 \mathrm{~nm}$ with a $570 \mathrm{~nm}$ long pass filter fitted. The machine was calibrated with standard fluorescent beads (Coulter UK) before use. A minimum of 15000 nuclei were analysed from each sample.

DNA histograms were interpreted in a standard manner. In each tumour sample stromal and inflammatory cell nuclei were present, providing an internal diploid standard. DNA aneuploidy was defined by the presence of a second $G_{0} / G_{1}$ peak which differed from the diploid $G_{0} / G_{1}$ channel number by at least $10 \%$. Ill defined "shoulders" on the diploid peak were not included as aneuploid peaks. A DNA index (defined as the ratio of the channel number of the aneuploid peak to the channel number of the diploid peak) was calculated for each tumour sample.

The routine archival (diagnostic) tissue blocks from each case in the study were obtained from the departmental file. From each block of malignant tissue a $50 \mu \mathrm{m}$ section was cut and processed for flow cytometry as described above. Examination by naked eye of the archival blocks showed that the amount of tissue contained in each was variable but, on the whole, was more than that contained in the blocks selected specifically for flow cytometry (fig 2). It was also obvious that many of the routine diagnostic blocks contained non-neoplastic lung tissue, or large areas of necrosis, or both. To prevent the presence of such elements masking the detection of aneuploidy in the tumour tissue, a further $50 \mu \mathrm{m}$ section was cut at a deeper level from each diagnostic block and a portion of tumour tissue free of these elements and equivalent in size to that selected for systematic sampling, was dissected out using a scalpel blade. The remaining tissue was discarded and the dissected tumour tissue processed for flow cytometry.

\section{Results}

The tumours were assigned to conventional histological groups as follows: squamous cell carcinoma ( $\mathbf{n}=10)$ : adenocarcinomas ( $\mathbf{n}=$ 4), small cell carcinoma $(n=3)$; and one each of large cell carcinoma, adenosquamous carcinoma, and atypical carcinoid tumour.

Systematic tumour sampling yielded a total of 208 tissue blocks (a mean of just over 10 per case). Examination of the routinely selected archival material showed that there were 84 blocks containing malignant tissue (a mean of four per case).

Table 1 shows the DNA analysis of the tissue obtained by systematic sampling: DNA aneuploid stemlines were detected in 19 of the $20(95 \%)$ cases. The DNA indices obtained varied from 1.2 to 2.5 . DNA indices in the tetraploid range (1.9-2.1) were identified in five cases. In each of these cases, however, other aneuploid stemlines (DNA indices $1 \cdot 2$, $1.6,1.3,3.0,1.5)$ were detected. The DNA abnormality was thus not merely a nuclear clumping effect. Heterogeneity of DNA content was shown in 18 carcinomas $(90 \%$ of the total). Of these cases, 12 contained a mixture of diploid and aneuploid areas; in six aneuploid stemlines of different DNA index were identified in separate areas of the tumour.

Table 1 Effect of sampling method in ploidy assessment in lung cancer

\begin{tabular}{|c|c|c|c|c|c|c|c|}
\hline \multirow[b]{3}{*}{ Case No } & \multirow[b]{3}{*}{ Histology } & \multicolumn{6}{|c|}{ Sampling method } \\
\hline & & \multicolumn{2}{|c|}{ Systematic } & \multicolumn{2}{|c|}{ Archival I } & \multicolumn{2}{|c|}{ Archival II } \\
\hline & & Diploid & Aneuploid & Diploid & Aneuploid & Diploid & Aneuploid \\
\hline 1 & Squamous & $1^{\star}$ & 4 & 0 & 2 & 0 & 2 \\
\hline 2 & Large cell & 0 & 10 & 0 & 3 & 0 & 3 \\
\hline 3 & Squamous & 0 & 10 & 0 & 3 & 0 & 3 \\
\hline 4 & Squamous & 10 & 0 & 4 & 0 & 4 & 0 \\
\hline 5 & Adeno & 0 & 10 & 1 & 5 & 0 & 5 \\
\hline 6 & Squamous & 0 & 10 & 0 & 3 & 0 & 3 \\
\hline 7 & Adeno & 0 & 10 & 0 & 6 & 0 & 6 \\
\hline 8 & Small cell $\dagger$ & 8 & 2 & 3 & 0 & 1 & 2 \\
\hline 9 & Squamous & 1 & 9 & 5 & 1 & $i$ & 5 \\
\hline 10 & Squamous & 1 & 9 & 2 & 1 & 1 & 2 \\
\hline 11 & Atypical carcinoid & 0 & 15 & 0 & 5 & 0 & 5 \\
\hline 12 & Adenosquamoust & 7 & 4 & 7 & 0 & 0 & 5 \\
\hline 13 & Adeno & 0 & 11 & 0 & 3 & 0 & 3 \\
\hline 14 & Squamoust & 5 & 11 & 5 & 0 & 0 & 4 \\
\hline 15 & Squamous & 9 & 3 & 3 & 1 & 1 & 2 \\
\hline 16 & Squamous & 0 & 10 & 2 & 5 & 1 & 4 \\
\hline 17 & Small cell & 7 & 3 & 0 & 4 & 0 & 4 \\
\hline 18 & Squamous ${ }^{\dagger}$ & 6 & 2 & 3 & 0 & 3 & 0 \\
\hline 19 & Small cell & 4 & 6 & 3 & 1 & 2 & 2 \\
\hline 20 & Adeno & 0 & 10 & 0 & 3 & 0 & 3 \\
\hline
\end{tabular}

*The figures refer to the number of tissue samples in each case.

tCases whose ploidy state was wrongly designated when initial sections were prepared from routine archival material (archival I). Only case 18 remained wrongly designated when tumour tissue was further selected (archival II). 
Only one case, the atypical carcinoid tumour, was homogeneously aneuploid.

On the other hand, when the initial tissue sections prepared from the routine archival blocks was analysed, aneuploidy was found in only 15 of the $20(75 \%)$ cases-that is, in four cases the routine sampling technique did not allow for proper assessment of the ploidy state of the tumour. Seventy seven of the 82 archival blocks contained sufficient tumour tissue to permit dissection of a second tumour sample. When these selected tumour areas were analysed by flow cytometry the number of cases containing aneuploid stemlines increased to $18(90 \%)$. Thus in only one case was the ploidy state of a tumour wrongly designated when this second method of tumour sampling was used. In all cases where aneuploidy was detected in the archival tissue (either by analysing the initial section of the full block or by selecting deeper tumour areas) the DNA index corresponded to that of a stemline detected by systematic sampling of the tumour.

\section{Discussion}

Since 1983, when Hedley et al first described a technique for recovering nuclei for flow cytometric DNA analysis from archival paraffin wax embedded tissue, ${ }^{9}$ there have been many papers published which describe the use of this technique for predicting the behaviour of a wide variety of tumours. Zimmerman et al published follow up data on a large series of surgically resected lung carcinomas and found that ploidy, determined by analysis of paraffin wax embedded tissue, was the most important predictor of prognosis on multivariate analysis. ${ }^{1}$ In his series $45 \%$ of the tumours were aneuploid. Other flow cytometric studies of lung cancer (based on analysis of fresh tissue) have shown variability in the percentage aneuploidy detected (80$96^{\circ}$ ), although even the lowest of these values is considerably higher than that reported by Zimmerman et al.

Table 2 summarises the data from all the major series reported to date. Although there are some differences in tumour stage and histological type among these series, the most important variation that might explain the observed differences is in the tumour sampling technique. The highest percentage aneuploidy reported $(96 \%)$ was obtained by analysing "multiple samples" (number unspecified) taken from both the tumour core and periphery. ${ }^{3}$ Interestingly this figure corresponds to the percentage of aneuploidy detec-

Table 2 Published reports showing variation in aneuploidy in lung cancer

\begin{tabular}{lll}
\hline Report & Method of sampling & $\begin{array}{l}\text { Percentage of } \\
\text { aneuploidy } \\
\text { detected }\end{array}$ \\
\hline Bunn et al $1983^{\circ}$ & Needle aspirates, lymph nodes, bronchial washings & 85 \\
Volm et al 1985" & Not stated & 82 \\
Tirindelli-Danesi et al & "Multiple samples" & 96 \\
Zimmerman et al 1987" & Archival & 45 \\
Van Bodegom et al $1989^{4}$ & Archival & 56 \\
\hline
\end{tabular}

ted in our study by systematic (multiple) sampling of tumours.

We have also found that only a small proportion (two out of 20 cases) of lung cancers show no heterogeneity within the tumour of DNA content. It must be assumed, therefore, that the variation in the percentage of aneuploidy reported in different series is a function of the differing sampling methods used; the more widely the tumour is sampled the greater the chances of detecting aneuploid stem lines. Indeed, if only one of the tumour samples taken by us in systematic sampling has been analysed in each case the possible percentage of aneuploidy could have ranged between $45-95 \%$-it is perhaps worth noting that these figures correspond to the range of values reported in the various series published so far.

As the percentage of aneuploidy detected in the different studies has been so variable it might be predicted that attempts to evaluate the prognostic importance of DNA content in lung cancers would yield conflicting results. This, in fact, has been the case. Early work by Bunn et al failed to show any association between ploidy and outcome of disease, ${ }^{10}$ while Volm et $a l^{2}$ and Zimmerman et $a l^{1}$ reported a strong correlation between the presence of aneuploidy in non-small cell lung cancer and a poor prognosis. Tirindelli-Danesi et al found that as only $4 \%$ of their tumours were diploid they were unable to make a valid comparison between diploid and aneuploid groups. ${ }^{3}$ These authors did, however, find that tumours with DNA stemlines of a DNA index of less than 1.0 or greater than 2.0 had a significantly poorer clinical outcome. More recently Van Bodegom et al claimed that the proportion of the total tumour cell population contained within the aneuploid peak was a better predictor of prognosis than the simple presence or absence of an abnormal stemline. ${ }^{4}$

Therefore, it seems likely that in the case of lung cancer, the tumour sampling technique is of cardinal importance in assessing the ploidy state. If flow cytometry is to be of use in predicting clinical outcome standardisation of sampling methods is imperative if relevant comparisons are to be made between different series. In our study wide sampling of tumour resection specimens showed that almost all lung cancers were aneuploid; only $75 \%$ of these same cases would have been so designated had routine archival blocks been used for DNA analysis. Dissection of tumour areas free of non-neoplastic or necrotic elements from the same blocks, however, improved the accuracy of sampling to over $90 \% .^{7}$ This method for avoiding sampling errors has been described in other tissues. ${ }^{11}$ We recommend that selection of tumour areas should always be performed when DNA analysis is undertaken on paraffin wax embedded material. Studies are in progress to assess the value of this method in predicting the clinical outcome in a large series of lung cancers.

We thank Eric Miller and Derek Bishop for their technical help and Helena Black who typed the manuscript. This work was partly funded by the Cancer Research Campaign. 
1 Zimmerman PV, Hawson GAT, Bint MH, Parsons PG. Ploidy as a prognostic determinant in surgically treated Ploidy as a prognostic determina

2 Volm M Drings P, Mattern J, et al. Prognostic significance of DNA patterns and resistance-predictive tests in nonsmall cell lung carcinoma. Cancer 1985;58:1396-403.

3 Tirindelli-Danesi D, Teodori L, Mauro F, et al. Prognostic significance of flow cytometry in lung cancer. Cancer 1987;60:844-51

4 Van Bodegom PC, Baak JPA, Stroet-Van Galen C, et al. The percentage of aneuploid cells is significantly correlated with survival in accurately staged patients with stage resected squamous cell lung cancer and long-term follow up. Cancer 1989;63:143-7.

5 Roggli VL, Vollmer RT, Greenberg SD, McGavran MH, Spjut HJ, Yesner R. Lung cancer heterogeneity: A blinded randomised study of 100 consecutive cases. Hum Pathol 1985;16:569-78.
6 Dunnill MS, Gatter KC. Cellular heterogeneity in lung cancer. Histopathol 1986;10:461-75.

7 Broers JLV, Rot MK, Oostendorp T, et al. Immunocytochemical detection of human lung cancer heterogeneity using antibodies to epithelial, neuronal and neuroendocrine antigens. Cancer Res 1987;47:3225-34.

8 Carey FA, Lamb D, Bird CC. Intratumoral heterogeneity of DNA content in lung cancer. Cancer 1990;65:2266-9.

9 Hedley DW, Friedlander ML, Taylor IW, et al. Method for analysis of cellular DNA content of paraffin-embedded pathological material using flow cytometry. I Histochem Cytochem 1983;31:1333-5.

10 Bunn PA, Carney DN, Gazdar AF, et al. Diagnostic and biological implications of flow cytometric DNA content biological implications of flow cytometric DNA content

11 Oud PS, Hanselaar TGJM, Reubsaet-Veldhuizen JAM, et al. Extraction of nuclei from selected regions in paraffin-embedded tissue. Cytometry 1986;7:595-600. 
squamous lesions, unusual tumours and tumour-like conditions, thyroid lesions in unusual places, cytology and needle biopsy and special techniques. The major emphasis is on histopathological diagnosis, supported by over 160 figures, mostly photomicrographs, over 50 tables and an enormous bibliography: there are 504 references to the chapter on medullary carcinoma alone.

The style is clear and readable. The author points out problems, cites opposing views and gently gives her own interpretation or viewpoint. The text is up to date, comprehensive, and satisfactorily illustrated. The recently described types of clear celled tumour, signet-ring carcinoma, insular carcinoma, hyalinising trabecular adenoma grooved nuclei in papillary carcinoma, are al there. A photomicrograph not included that would have been helpful is an example of capsular invasion which the author considers diagnostic of malignancy in follicular tumours, even in the absence of vascular invasion or complete capsular transgression. Dr LiVolsi is to be congratulated on this excellent monograph. It should be on the bookshelf in every laboratory responsible for service histopathology.

I DONIACH

Principles of Drug Action. The Basis of Pharmacology. 3rd ed. Ed WB Pratt, P Taylor. (Pp 816; $£ 37.50$.) Churchill Living stone. 1990. ISBN 0-443-08676-1.

The organisation of most pharmacology texts is based on organ systems, classes of drugs, or disease states. In this easily readable but detailed book a novel approach is taken. The biological, chemical, and molecular concepts which are the basis of pharmacology and thus underlie the principles of drug action are presented.

The opening chapters cover the molecular basis of drug specificity, and this is one of the few general pharmacology books to include information on the influence of chirality and stereoselectivity on the interaction between the drug molecule and its site of action. Many examples in which an unusual response to a drug may be precipitated by hereditary factors are given in the chapter on pharmacogenetics. This book also covers the areas of carcinogenesis, mutagenesis, and teratogenesis in addition to drug metabolism, allergy, resistance, tolerance, and physical dependence.

There have been tremendous advances in the understanding of drug action in the 16 years since the last edition of this textbook and, with the advent of new technology that knowledge is still evolving. My major criticism is that textbooks which include state-ofthe-art research in a rapidly advancing area will quickly become out of date. It is to be hoped that we do not have to wait another 16 years for the next revision. There is a definite need for a frequently updated text of this kind available at a reasonable price.

In short, this is a book which will help not only the pharmacologist, but also the biological scientist, chemist, and clinician to understand the factors which regulate and determine drug action.
Advanced Histopathology. GWH Stamp, NA Wright. (Pp 350; soft cover DM60.) Springer. 1990. ISBN 3-540-19589-0.

The arrival of an "Aids to MRCPath" book has been long awaited by histopathology trainees such as myself for whom the final exam looms large on the horizon. Advanced Histopathology admirably fills this gap in the market. Contrary to what its title may suggest, this is not a conventional textbook of pathology; rather, it is a trainee's vademecum aimed specifically at how to pass the MRCPath.

The book is divided into sections covering all aspects of the examination including the written paper, post mortem, practical, and viva voce. The largest section of the book (242 pages) is devoted to the written exam. Papers have been reviewed back to 1969 and specimen answers are illustrated. About one quarter of the answers are in the form of essay plans, the remainder as explanatory paragraphs. The post mortem and practical sections are reviewed in slightly less detail, although this is inevitable given the variability of the exam from centre to centre.

The style of the book is informal rather than didactic and I found it very readable One minor criticism is that for many candidates at this stage of their career, much of the information is superfluous; how many of us need to be reminded to take an extra pen to the exam in case the one we're using runs out? This aside, the book is helpful and informative and will, I believe, help most candidates optimise their approach to this formidable exam.

P DOMIZIO

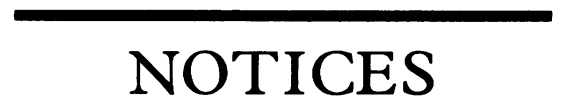

\begin{tabular}{l} 
Association of Clinical Pathologists \\
Junior Membership \\
Tunior membership of the Association is \\
available to medical practitioners who \\
have been engaged in the practice of \\
pathology for a period of less than four \\
years. Junior members are able to remain \\
in this category for a maximum of six years \\
or on the attainment of consultant status. \\
The annual subscription is $£ 24$ for those \\
resident in the United Kingdom and $£ 55$ \\
for those overseas. The annual subscrip- \\
tion may be claimed against tax. \\
Junior members receive the Journal of \\
Clinical Pathology each month. Other \\
benefits are reduced registration fees to \\
attend ACP scientific meetings, all the \\
documents regularly sent to full members \\
of the Association including ACP News, \\
which has a regular column for juniors, \\
and the twice yearly summary of path- \\
ology courses included in the ACP \\
programme of postgraduate education. \\
Junior members have their own \\
representative body, the Junior Members' \\
Group, which has a direct input to Coun- \\
cil. \\
For Junior Membership apply to: The \\
Honorary Secretary, Association of \\
Clinical Pathologists, School of Biological \\
Sciences, Falmer, Brighton, BN1 9QG. \\
(0273) 678435. \\
\hline
\end{tabular}

\section{ACP Locum Bureau}

The Association of Clinical Pathologists runs a locum bureau for consultant pathologists.

Applicants with the MRCPath who would like to do locums and anyone requiring a locum should contact The General Secretary, School of Biological Sciences, Falmer, Brighton, BN1 9QC Tel and Fax: 0273678435

\section{Corrections}

We are indebted to Dr Hatchérian of Fresnes for pointing out some errors in units given in the article "Thrombotic thrombocytopenic purpura (TTP) complicating leptospirosis" (1990;43:961). The fibrinogen concentration should have been expressed as $\mathrm{g} / \mathrm{l}$ throughout (not $\mathrm{g} / \mathrm{dl}$ as it was in one instance), and the bilirubin should have been indicated to be $101 \mu \mathrm{mol}$ not $101 \mathrm{mmol}$. The editors try to be vigilant about such things, but slip-ups occur occasionally and we are grateful to the readers who keep us on our toes.

An error appeared in the paper, Importance of sampling method in DNA analysis of lung cancer $(1990 ; 43: 820-3)$ : in the second to last line of the abstract tumour selection should have been printed rather than turnover selection. 